\title{
Weiterbildung aus der Sicht der Assistenzärzte
}

\author{
Die wichtigsten Resultate der Umfrage 2004 bei Assistenzärztinnen und -ärzten über die Weiterbildung
}

\author{
M. Siegrist ${ }^{a}$, P. Orlow ${ }^{a}$, M. Giger $^{b}$
}

Zum siebten Mal wurde die Umfrage zur Weiterbildung bei den Assistenzärztinnen und Assistenzärzten, welche einen eidgenössischen Weiterbildungstitel anstreben, durchgeführt. Die Rücklaufquote beträgt $65 \%$. Ein Viertel der Assistenzärztinnen und Assistenzärzte sind im Besitz eines deutschen, knapp zwei Drittel eines schweizerischen Arztdiploms. Im Verlaufe eines Jahres wurde die wöchentliche Arbeitszeit in allen Fachgebieten reduziert. Sie wird mit 59 Stunden von den chirurgischen Weiterbildungsstätten angeführt. 94\% der Weiterbildungsstätten wurden positiv beurteilt. Die Fehlerkultur ist in der Pädiatrie und Anästhesiologie am besten eingeführt. Sie sollte in allen Fachgebieten in der Weiterbildung ausgebaut werden. In der Psychiatrie zeichnet sich eine deutliche Abnahme der Facharzttitelträger ab.

\section{a Sozialforschungsstelle der Universität Zürich \\ b Ressort Medical Education FMH}

1 Daugherty SR, Baldwin DC, Rowley BD. Learning, satisfaction, and mistreatment during medical internship: a national survey of working conditions. JAMA 1998; 279:1194-9.

2 Blumenthal D, Gokhale M, Campbell EG, Weissman JS. Preparedness for clinical practice: reports of graduating residents at academic health centers. JAMA 2001;286:1027-34.

3 Sibbald B, Enzer I, Cooper C, Rout U, Sutherland V. GP job satisfaction in 1987, 1990 and 1998: lessons for the future? Fam Pract 2000;17:364-71.

4 Goldacre MJ, Lambert T, Evans J, Turner G. Preregistration house officers' views on whether their experience at medical school prepared them well for their jobs: national questionnaire survey. BMJ 2003;326:1011-2.

(Literaturhinweise 5 und 6 auf der nächsten Seite)

Korrespondenz:

PD Dr. med. Michael Siegrist

Sozialforschungsstelle

Universität Zürich,

Plattenstrasse 14

CH-8032 Zürich
Die jährliche Befragung der Assistenzärzte liefert Informationen über die Weiterbildungsqualität der Weiterbildungsstätten. Der Vorteil einer relativ effizienten Datenbeschaffung ist dabei mit dem Nachteil einer subjektiven Beurteilung verknüpft. Aus ökonomischen Gründen gibt es in der Medizin aber eine Tradition, Qualitätsbeurteilungen aufgrund von Daten aus Befragungen vorzunehmen [1-4].

Die Umfrage 2003 bei den Assistenzärzten machte deutlich, dass die globale Beurteilung einer Weiterbildungsstätte stark durch weiche Faktoren beeinflusst wird [5, 6]. Gutes Arbeitsund Führungsklima sowie die wahrgenommene Qualität der Weiterbildung bestimmten in hohem Masse die Globalbeurteilung einer Weiterbildungsstätte. Die Daten machten deutlich, dass strukturelle Faktoren einen Einfluss auf die Wahrnehmung einer Weiterbildungsstätte haben. Bei den Fächern mit den meisten Assistenzärzten schnitten 2003 die Chirurgie schlecht und die Anästhesiologie gut ab. Weiterbildungsstätten mit vielen Assistenzärzten wurden tendenziell negativer beurteilt als Weiterbildungsstätten mit wenigen Assistenzärzten.

Die siebte Umfrage zur Weiterbildung unter den Assistenzärzten erfolgte in den Monaten Juli bis Oktober 2004. Die Entwicklung des Fragebogens wurde bei der Präsentation der Ergebnisse aus dem Jahre 2003 ausführlich beschrieben $[5,6]$. Die Leiterinnen und Leiter von Weiterbildungsstätten erhielten eine detaillierte Rückmel- dung mit den Ergebnissen ihrer Weiterbildungsstätte und Vergleichswerten von den anderen Weiterbildungsstätten der entsprechenden Fachrichtung.

\section{Methode}

\section{Stichprobe}

Die Fragebogen wurden an 1212 Leiter von Weiterbildungsstätten geschickt. Diese waren für die Verteilung an die 8229 Assistenzärzte verantwortlich. Die ausgefüllten Fragebogen konnten die Assistenzärzte direkt an die Sozialforschungsstelle der Universität Zürich schicken, welche für die Durchführung der Befragung verantwortlich war. Die Rücklaufquote betrug $65 \%(\mathrm{n}=5388)$. Dieser Wert ist vergleichbar mit der Rücklaufquote der Umfrage 2003 [5]. $47 \%$ der Fragebogen sind von Assistenzärztinnen und 53\% von Assistenzärzten ausgefüllt worden.

Lagen von einer Weiterbildungsstätte mindestens vier ausgefüllte Fragebogen vor, so erhielt der Leiter der Weiterbildungsstätte eine Rückmeldung mit den Durchschnittswerten. Die Assistenzärzte konnten im Fragebogen angeben, ob ihre Daten benutzt werden dürfen, wenn weniger als vier ausgefüllte Fragebogen vorliegen. Sind die Untersuchungseinheiten Weiterbildungsstätten, werden im folgenden dieselben Daten wie zur Rückmeldung an die Leiter der Weiterbildungsstätten benutzt. Sind die Untersuchungseinheiten dagegen Assistenzärzte, so werden alle ausgefüllten Fragebogen berücksichtigt. Für die Beurteilung der Weiterbildungsstätten werden nur die Antworten der Assistenzärzte berücksichtigt, welche mindestens zwei Monate an der entsprechenden Stelle tätig waren.

\section{Fragebogen}

Der Fragebogen enthielt 80 Items. In erster Linie mussten die Befragten zu Aussagen Stellung nehmen. Dabei standen bei den meisten Items sechs Antwortkategorien zur Auswahl, von 1 «trifft überhaupt nicht zu» bis zu 6 «trifft voll und ganz zu». 
Der Fragebogen der Umfrage 2003 [6] wurde überarbeitet. Ein Teil der Fragen wurde umformuliert oder ersetzt. Für die Themenfelder Umgang mit Fehlern und Evidence-based Medicine wurden die Items gänzlich neu formuliert. Zudem wurde im Fragebogen 2004 das Modul Attitude berücksichtigt. Bei diesem Themenblock geht es um die Frage, ob an der Weiterbildungsstätte auch ethische Prinzipien vermittelt werden und ob die Assistenzärzte angeleitet werden, Selbstverantwortung zu übernehmen.

Aufgrund von theoretischen und statistischen Überlegungen wurden die Aussagen zu Skalen zusammengefasst, die im folgenden beschrieben werden. Für die Abschätzung der Skalengüte wurde Cronbachs Alpha verwendet. Dieser Koeffizient kann Werte zwischen 0 und 1 annehmen. Je höher der Wert, desto grösser ist die interne Konsistenz einer Skala. Mit anderen Worten, die Messgenauigkeit der Skala ist höher. Die Reliabilitätskoeffitienten und beispielhafte Fragen sind in Tabelle 1 aufgeführt.

Aufgrund der Skalenwerte wurde für jede Weiterbildungsstätte ein Kennwert berechnet. Dabei wurden die Dimensionen Lernkultur und Weiterbildungsziele mit drei und die Dimension Evidence-based Medicine mit zwei gewichtet. Die restlichen fünf Dimensionen wurden einfach gewichtet (Globalbeurteilung, Führungskultur, Fehlerkultur, Entscheidungskultur, Betriebskultur). Durch diese Gewichtung wurden die für die Weiterbildung besonders zentralen Dimensionen auch entsprechend gewichtet. Bei fehlenden Werten wurde die Gewichtung entsprechend angepasst, so dass die Kennwerte möglichst gut vergleichbar sind.
Als zusätzliche Dimensionen wurden mit vier Items die Motivation $(\alpha=0,76)$ und mit drei Items der Stress $(\alpha=0,58)$ gemessen. Als jährliches Modul wurden in der Umfrage 2004 Fragen zur Attitude gestellt (z.B. auf Respekt und Ehrlichkeit gegenüber dem Patienten wird an unserer Weiterbildungsstätte grosses Gewicht gelegt). Die fünf Fragen konnten zu einer reliablen Skala zusammengefasst werden $(\alpha=0,81)$.

\section{Ergebnisse}

Die Antworten auf die Frage nach dem angestrebten Facharzttitel sind in Tabelle 2 dargestellt. An der starken Feminisierung in den Richtungen Anästhesiologie, Gynäkologie, Kinder- und Jugendmedizin sowie Kinder- und Jugendpsychiatrie hat sich 2004 wenig geändert. In der Richtung Psychiatrie und Psychotherapie nahm der Frauenanteil 2004 im Vergleich zum Vorjahr sogar deutlich zu.

Von den befragten Assistenzärzten absolvierten $76,9 \%(n=4095)$ ein fachspezifisches Jahr und $14,8 \%(n=790)$ ein Fremdjahr. 8,3\% $(n=442)$ hatten sich noch nicht für einen Facharzttitel entschieden.

Die Kohorten mit Staatsexamen 1996 bis und mit Staatsexamen 2003 wurden miteinander verglichen. Weil nur wenige Assistenzärzte mit dem Staatsexamen 2004 den Fragebogen ausgefüllt hatten und die Kohorten 1996 oder früher deutlich kleiner waren, wurden diese für die folgenden Analysen nicht berücksichtigt. Die Ergebnisse in Tabelle 3 zeigen, dass ein grosser

Tabelle 1

Die wichtigsten Skalen.

5 Giger M, Siegrist M. Ärztliche Weiterbildung auf dem Prüfstand. Schweiz Ärztezeitung 2003;84(50): 2655-7.

6 Siegrist M, Gutscher H, Giger M. Was haben Betriebskultur, Motivation und Fremdjahr mit der Weiterbildung zu tun? Schweiz Ärztezeitung 2004;85(15):783-91.

\begin{tabular}{llcc}
\hline Skala & Beispiel für Items & Anzahl Items & Alpha \\
\hline Globalbeurteilung & Ich würde die Weiterbildungsstätte weiterempfehlen & 3 & 0,92 \\
\hline Lernkultur & $\begin{array}{l}\text { Ich erhalte regelmässig Rückmeldungen darüber, was ich richtig } \\
\text { und was ich falsch gemacht habe }\end{array}$ & 13 & 0,92 \\
\hline Führungskultur & Meine Vorgesetzten sorgen für eine gute Arbeitsorganisation & 5 & 0,87 \\
\hline Fehlerkultur & $\begin{array}{l}\text { An unserer Weiterbildungsstätte gibt es regelmässig Konferenzen } \\
\text { zur Besprechung von Komplikationen und Fehlern }\end{array}$ & 3,68 \\
\hline Entscheidungskultur & $\begin{array}{l}\text { Ich lerne, dass bei einer medizinischen Entscheidung auch } \\
\text { das soziale Umfeld einbezogen werden muss }\end{array}$ & 2 & 0,89 \\
\hline $\begin{array}{l}\text { Weiterbildungsziele } \\
\text { Betriebskultur }\end{array}$ & $\begin{array}{l}\text { Ich habe die vereinbarten Ziele zu 100\% erreicht } \\
\text { In unserer Weiterbildungsstätte (Ärzte und Pflegepersonal) } \\
\text { gehen wir ehrlich und direkt miteinander um }\end{array}$ & 2 & 4 \\
\hline $\begin{array}{l}\text { Evidence-based } \\
\text { Medicine }\end{array}$ & $\begin{array}{l}\text { Ich lerne, die Ergebnisse einer wissenschaftlichen Studie konkret } \\
\text { bei der Behandlung eines Patienten anzuwenden }\end{array}$ & 4 \\
\hline $\begin{array}{l}\text { Anmerkung: Nur die Antworten von Assistenzärzten, die zwei Monate oder länger an der Weiterbildungsstätte } \\
\text { tätig waren, wurden berücksichtigt. }\end{array}$
\end{tabular}


Tabelle 2

Angestrebter Facharzttitel getrennt nach Geschlecht für die Umfragen 2003 und 2004.

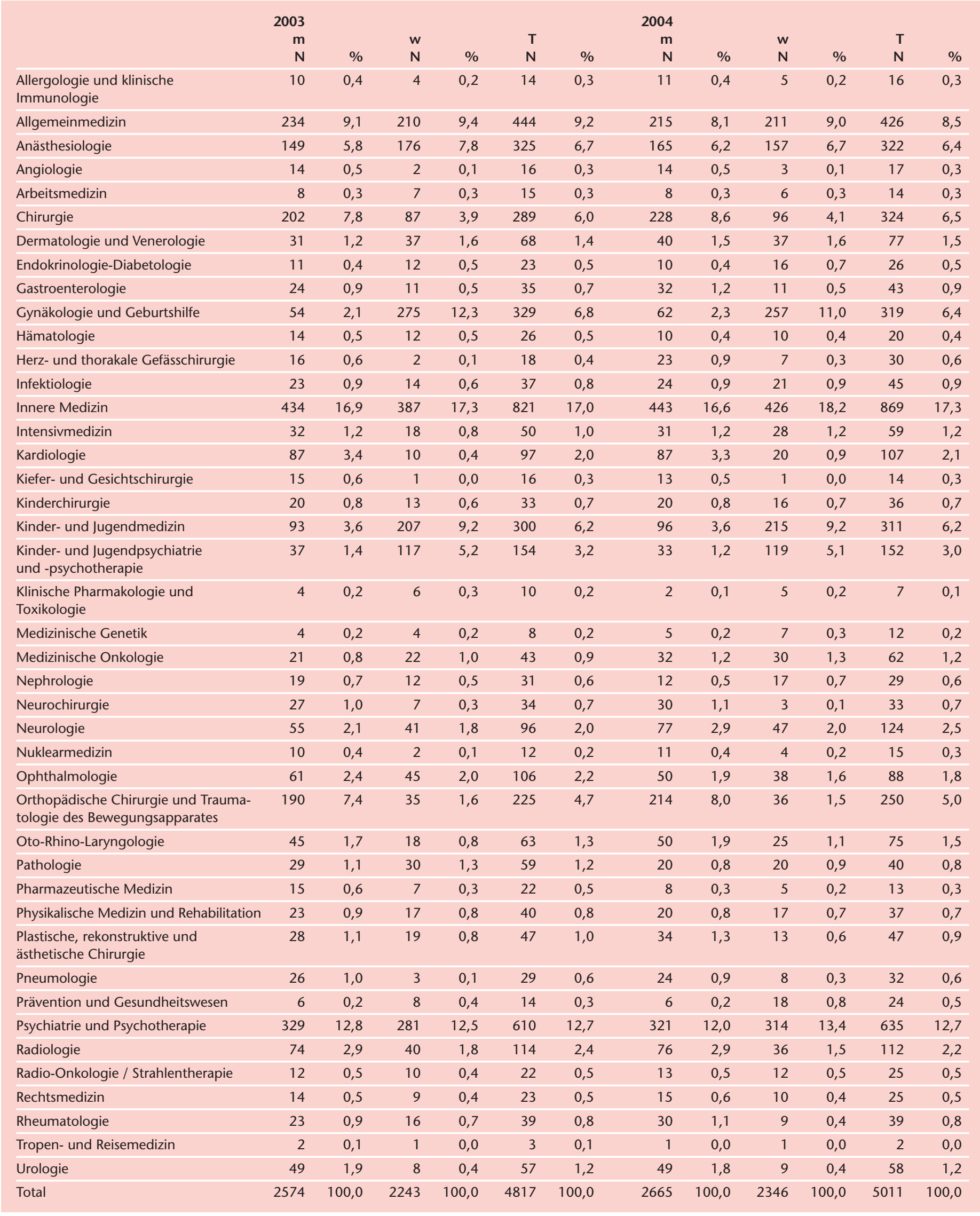


Teil der Assistenzärzte schon relativ früh weiss, welchen Facharzttitel sie anstreben möchten.

Tabelle 4 zeigt die angestrebten Facharzttitel für die Kohorten 1998 bis 2003. Die weiter zurückliegenden Kohorten wurden nicht berücksichtigt, weil ein Teil der Assistenzärzte bereits einen Facharzttitel hat und deshalb in der Stichprobe nicht mehr vertreten ist. Kaum relative Unterschiede zwischen den Kohorten gibt es für die Allgemeinmedizin, die Gynäkologie, Kinderund Jugendmedizin und Orthopädische Chirurgie. Ein ausgeprägter Trend konnte aber für die Psychiatrie und Psychotherapie beobachtet werden. Die relative Zahl der Assistenzärzte, welche diesen Titel anstreben, ist in der Kohorte 2003 nur noch halb so gross wie in der Kohorte 1998. Der umgekehrte Trend konnte dagegen in der Chirurgie beobachtet werden. Der relative Anteil an Chirurgen ist bei der jüngeren Kohorte deutlich grösser als bei der älteren Kohorte.

Tabelle 3

Die Kohorten mit Abschluss Staatsexamen 1996 bis 2003 und Entscheidung für Facharzttitel.

\begin{tabular}{|c|c|c|c|}
\hline & \multicolumn{2}{|c|}{ Für Facharzttitel entschieden } & \multirow[t]{2}{*}{ Total } \\
\hline & Ja & Nein & \\
\hline 1996 & $96,1 \%(n=269)$ & $3,9 \%(n=11)$ & $100 \%(n=280)$ \\
\hline 1997 & $98,9 \%(n=360)$ & $1,1 \%(n=4)$ & $100 \%(n=364)$ \\
\hline 1998 & $97,8 \%(n=490)$ & $2,2 \%(n=11)$ & $100 \%(n=501)$ \\
\hline 1999 & $97,3 \%(n=581)$ & $2,7 \%(n=16)$ & $100 \%(n=597)$ \\
\hline 2000 & $94,1 \%(n=571)$ & $5,9 \%(n=36)$ & $100 \%(n=607)$ \\
\hline 2001 & $92,0 \%(n=657)$ & $8,0 \%(n=57)$ & $100 \%(n=714)$ \\
\hline 2002 & $87,8 \%(n=590)$ & $12,2 \%(n=82)$ & $100 \%(n=672)$ \\
\hline 2003 & $75,3 \%(n=472)$ & $24,7 \%(n=155)$ & $100 \%(n=627)$ \\
\hline
\end{tabular}

Von den Befragten hatten $64,7 \%$ das Staatsexamen in der Schweiz gemacht und $25,4 \%$ in Deutschland. In der Umfrage 2003 hatten noch 22,6\% der Befragten Deutschland angegeben. Zwischen den drei Sprachregionen konnten deutliche Unterschiede beobachtet werden (Tab. 5). Der relative Anteil von Assistenzärzten, welche das Staatsexamen in der Schweiz gemacht hatten, ist in der Westschweiz am grössten. Jeder dritte Assistenzarzt, der in der Deutschschweiz arbeitet, hat sein Staatsexamen in Deutschland gemacht.

Der Tabelle 6 kann entnommen werden, welcher Facharzttitel von Personen angestrebt wird, die das Staatsexamen in der Schweiz oder im Ausland gemacht haben. Bei den Anwärtern für den Facharzttitel Orthopädische Chirurgie und Traumatologie des Bewegungsapparates sowie Psychiatrie und Psychotherapie dominieren ganz deutlich die Assistenzärzte, welche das Staatsexamen im Ausland bestanden haben.

Der Zusammenhang zwischen dem angestrebten Facharzttitel und der Fachrichtung der Weiterbildungsstätte, an welcher die Assistenzärzte zur Zeit der Befragung arbeiteten, kann Tabelle 7 entnommen werden. Die Zeilenprozente zeigen, dass nur 9\% der Assistenzärzte, welche den Facharzttitel Psychiatrie und Psychotherapie anstreben, in der Befragungsperiode ein Fremdjahr absolvierten. Ein Weiterbildungsjahr in psychiatrischen Weiterbildungsstätten wird am häufigsten von den Allgemeinmedizinern, gefolgt von den Internisten absolviert. Assistenzärzte, die sich noch nicht für einen Facharzttitel entschieden haben, arbeiten am häufigsten an einer Weiterbildungsstätte des Fachgebiets Innere Me-

Tabelle 4

Die Kohorten mit Abschluss Staatsexamen 1998 bis 2003 und die angestrebten Facharzttitel.

\begin{tabular}{|c|c|c|c|c|c|c|}
\hline & 1998 & 1999 & 2000 & 2001 & 2002 & 2003 \\
\hline Allgemeinmedizin & $7,7 \%(n=39)$ & $8,4 \%(n=50)$ & $8,7 \%(n=53)$ & $10,3 \%(n=74)$ & $8,3 \%(n=56)$ & $8,3 \%(n=52)$ \\
\hline Anästhesiologie & $7,7 \%(n=39)$ & $7,5 \%(n=45)$ & $6,4 \%(n=39)$ & $5,7 \%(n=41)$ & $8,3 \%(n=56)$ & $6,1 \%(n=38)$ \\
\hline Chirurgie & $5,1 \%(n=26)$ & $4,4 \%(n=26)$ & $5,9 \%(n=36)$ & $7,7 \%(n=55)$ & $8,8 \%(n=59)$ & $10,5 \%(n=66)$ \\
\hline Gynäkologie & $7,7 \%(n=39)$ & $7,9 \%(n=47)$ & $4,6 \%(n=28)$ & $6,7 \%(n=48)$ & $6,1 \%(n=41)$ & $7,2 \%(n=45)$ \\
\hline Innere Medizin & $16,2 \%(n=82)$ & $20,6 \%(n=123)$ & $19,2 \%(n=117)$ & $21,5 \%(n=154)$ & $20,7 \%(n=139)$ & $13,6 \%(n=85)$ \\
\hline $\begin{array}{l}\text { Kinder- und } \\
\text { Jugendmedizin }\end{array}$ & $5,9 \%(n=30)$ & $7,7 \%(n=46)$ & $7,4 \%(n=45)$ & $6,9 \%(n=49)$ & $6,4 \%(n=43)$ & $7,0 \%(n=44)$ \\
\hline $\begin{array}{l}\text { Orthopädische Chirurgie } \\
\text { und Traumatologie } \\
\text { des Bewegungsapparates }\end{array}$ & $5,3 \%(n=27)$ & $4,5 \%(n=27)$ & $5,4 \%(n=33)$ & $5,3 \%(n=38)$ & $4,9 \%(n=33)$ & $6,9 \%(n=43)$ \\
\hline $\begin{array}{l}\text { Psychiatrie und } \\
\text { Psychotherapie }\end{array}$ & $11,1 \%(n=56)$ & $10,9 \%(n=65)$ & $11,5 \%(n=70)$ & $7,8 \%(n=56)$ & $5,6 \%(n=38)$ & $4,9 \%(n=31)$ \\
\hline Anderes & $33,1 \%(n=167)$ & $28,1 \%(n=168)$ & $31,0 \%(n=189)$ & $28,0 \%(n=200)$ & $30,9 \%(n=208)$ & $35,6 \%(n=223)$ \\
\hline
\end{tabular}


dizin und am zweithäufigsten an einer Weiterbildungsstätte des Fachbereichs Chirurgie.

Die Kolonnenprozente in Tabelle 7 zeigen, wie viele fachfremde Assistenzärzte in den verschiedenen Weiterbildungsstätten tätig sind. Die Weiterbildungsstätten Chirurgie weisen den höchsten und die Weiterbildungsstätten Kinderund Jugendmedizin weisen den tiefsten Wert auf.

\section{Neuer Kennwert}

Der im Methodikteil beschriebene Kennwert wird benutzt, um Weiterbildungsstätten zu identifizieren, die bei der Beurteilung durch die Assistenzärzte schlecht abgeschnitten haben. Im folgenden wird deshalb noch genauer untersucht, welche strukturellen Faktoren einen Einfluss auf den Kennwert hatten. Zwischen den Fachrichtungen konnten systematische Unterschiede für den Kennwert beobachtet werden. Die Ergebnisse für die sieben Fachrichtungen mit den grössten Assistentenzahlen sind in Tabelle 8 aufgeführt. Die Chirurgie wies dabei einen signifikant tieferen Wert auf als die anderen Fachrichtungen. In dieser Form wurde der Kennwert 2004 zum ersten Mal berechnet. Ein Vergleich mit früheren Jahren ist deshalb nur beschränkt möglich. In der der Umfrage 2003 wurde die Globalbeurteilung relativ umfassend beschrieben [6]. Vergleicht man die Globalbeurteilung für die sieben Fachrichtungen mit den meisten Assistenzärzten, so ergeben sich keine signifikanten Unterschiede zwischen den Resultaten der Umfrage 2003 und der Umfrage 2004.

Die Grösse der Weiterbildungsstätte hat zum Teil einen Einfluss darauf, wie diese beurteilt wird. Die Mittelwerte für den Kennwert aufgeschlüsselt nach Grösse der Weiterbildungsstätte und Fachrichtung sind in Tabelle 9 aufgeführt. Für den Kennwert spielte die Grösse der Weiterbildungsstätte lediglich in den Fachrichtungen

Tabelle 5

Land, in dem Staatsexamen abgeschlossen wurden, aufgeschlüsselt nach Sprachregion, in welcher die Weiterbildungsstätte liegt (Kolonnenprozente und absolute Zahlen).

\begin{tabular}{lllll} 
& \multicolumn{1}{l}{$\begin{array}{l}\text { Region } \\
\text { Deutschschweiz }\end{array}$} & Westschweiz & Tessin & Total \\
\hline Schweiz & $61,0 \%(n=2282)$ & $77,6 \%(n=1084)$ & $40,5 \%(n=70)$ & $64,7 \%(n=3436)$ \\
\hline Deutschland & $33,4 \%(n=1251)$ & $5,8 \%(n=81)$ & $10,4 \%(n=18)$ & $25,4 \%(n=1350)$ \\
\hline Frankreich & $0,1 \%(n=5)$ & $1,6 \%(n=22)$ & $1,2 \%(n=2)$ & $0,5 \%(n=29)$ \\
\hline Italien & $0,2 \%(n=6)$ & $1,5 \%(n=21)$ & $37,6 \%(n=65)$ & $1,7 \%(n=92)$ \\
\hline Österreich & $2,0 \%(n=76)$ & $0,4 \%(n=6)$ & $0,0 \%(n=0)$ & $1,5 \%(n=82)$ \\
\hline Restliche EU & $1,0 \%(n=37)$ & $4,4 \%(n=61)$ & $0,0 \%(n=0)$ & $1,8 \%(n=98)$ \\
\hline Anderes & $2,3 \%(n=85)$ & $8,7 \%(n=122)$ & $10,4 \%(n=18)$ & $4,2 \%(n=225)$
\end{tabular}

Tabelle 6

Die angestrebten Facharzttitel der in der Deutschschweiz tätigen Assistenzärzte, aufgeschlüsselt nach Staatsexamen, in der Schweiz oder im Ausland gemacht.

\begin{tabular}{llll} 
& Schweiz & Ausland & Total \\
\hline Allgemeinmedizin & $67,5 \%(n=204)$ & $32,5 \%(n=98)$ & $100 \%(n=302)$ \\
\hline Anästhesiologie & $75,2 \%(n=170)$ & $24,8 \%(n=56)$ & $100 \%(n=226)$ \\
\hline Chirurgie & $53,5 \%(n=138)$ & $46,5 \%(n=120)$ & $100 \%(n=258)$ \\
\hline Gynäkologie & $54,6 \%(n=131)$ & $45,4 \%(n=109)$ & $100 \%(n=240)$ \\
\hline Innere Medizin & $69,6 \%(n=431)$ & $30,4 \%(n=188)$ & $100 \%(n=619)$ \\
\hline Kinder- und Jugendmedizin & $82,4 \%(n=155)$ & $17,6 \%(n=33)$ & $100 \%(n=188)$ \\
\hline Kinder- und Jugendpsychiatrie & $54,0 \%(n=47)$ & $46,0 \%(n=40)$ & $100 \%(n=87)$ \\
\hline $\begin{array}{l}\text { Orthopädische Chirurgie und Traumatologie } \\
\text { des Bewegungsapparates }\end{array}$ & $34,4 \%(n=65)$ & $65,6 \%(n=124)$ & $100 \%(n=189)$ \\
\hline Psychiatrie und Psychotherapie & $39,1 \%(n=158)$ & $60,9 \%(n=246)$ & $100 \%(n=404)$ \\
\hline Anderes & $63,7 \%(n=783)$ & $36,3 \%(n=446)$ & $100 \%(n=1229)$ \\
\hline
\end{tabular}


Anästhesiologie, Innere Medizin sowie Gynäkologie und Geburtshilfe eine Rolle. Weiterbildungsstätten mit wenigen Assistenten wurden positiver beurteilt als solche mit vielen Assistenten.

In Tabelle 10 sind die Durchschnittswerte des Kennwertes auf der Ebene der Weiterbildungsstätten dargestellt. Insgesamt wurden die Weiterbildungsstätten sehr positiv beurteilt. Es gibt aber Weiterbildungsstätten, bei denen ein Verbesserungspotential vorhanden ist. So weisen $6,4 \%$ der Weiterbildungsstätten einen Wert von 3,5 oder weniger auf.

\section{Attitude}

Für das Konstrukt Attitude konnten relativ hohe Mittelwerte beobachtet werden. In vielen Weiterbildungsstätten haben die Assistenzärzte also den Eindruck, dass ethische Prinzipien vermittelt werden, dass auf Respekt und Ehrlichkeit gegenüber dem Patienten Gewicht gelegt wird und dass die Assistenzärzte angehalten werden, Verantwortung zu übernehmen. Es konnten aber systematische Unterschiede zwischen den einzelnen Fachrichtungen beobachtet werden (vgl. Tab. 11). Die Fachrichtung Psychiatrie und Psychotherapie hatte einen signifikant höheren Mittelwert als die anderen Fachrichtungen.

Tabelle 7

Angestrebter Facharzttitel und Fachrichtung der Weiterbildungsstätte (Zeilenprozente, Kolonnenprozente und absolute Zahlen).

\begin{tabular}{|c|c|c|c|c|c|c|c|c|}
\hline \multirow[t]{2}{*}{ Angestrebter Facharzttitel } & \multicolumn{8}{|c|}{ Weiterbildungsstätte } \\
\hline & $\begin{array}{l}\text { Innere } \\
\text { Medizin }\end{array}$ & $\begin{array}{l}\text { Anästhesio- } \\
\text { logie }\end{array}$ & Chirurgie & Gynäkologie & $\begin{array}{l}\text { Kinder- und } \\
\text { Jugendmedizin }\end{array}$ & Psychiatrie & $\begin{array}{l}\text { Orthopädische } \\
\text { Chirurgie }\end{array}$ & Andere \\
\hline \multirow[t]{3}{*}{ Allgemeinmedizin } & $43,3 \%$ & $1,2 \%$ & $19,4 \%$ & $6,8 \%$ & $1,6 \%$ & $8,7 \%$ & $1,4 \%$ & $17,6 \%$ \\
\hline & $14,3 \%$ & $1,9 \%$ & $11,9 \%$ & $8,7 \%$ & $2,6 \%$ & $5,1 \%$ & $3,1 \%$ & $4,7 \%$ \\
\hline & $(n=185)$ & $(n=5)$ & $(n=83)$ & $(n=29)$ & $(n=7)$ & $(n=37)$ & $(n=6)$ & $(n=75)$ \\
\hline \multirow[t]{3}{*}{ Anästhesiologie } & $14,3 \%$ & $67,1 \%$ & $5,0 \%$ & & $0,3 \%$ & $0,3 \%$ & $0,6 \%$ & $12,4 \%$ \\
\hline & $3,5 \%$ & $82,4 \%$ & $2,3 \%$ & & $0,4 \%$ & $0,1 \%$ & $1,0 \%$ & $2,5 \%$ \\
\hline & $(n=46)$ & $(n=216)$ & $(n=16)$ & & $(n=1)$ & $(n=1)$ & $(n=2)$ & $(n=40)$ \\
\hline \multirow[t]{3}{*}{ Chirurgie } & $3,4 \%$ & $0,6 \%$ & $89,0 \%$ & $0,3 \%$ & & & $2,1 \%$ & $4,6 \%$ \\
\hline & $0,8 \%$ & $0,8 \%$ & $41,8 \%$ & $0,3 \%$ & & & $3,6 \%$ & $0,9 \%$ \\
\hline & $(n=11)$ & $(n=2)$ & $(n=291)$ & $(n=1)$ & & & $(n=7)$ & $(n=15)$ \\
\hline \multirow[t]{3}{*}{ Gynäkologie } & $0,3 \%$ & $0,6 \%$ & $9,4 \%$ & $89,0 \%$ & $0,3 \%$ & & & $0,3 \%$ \\
\hline & $0,1 \%$ & $0,8 \%$ & $4,3 \%$ & $85,3 \%$ & $0,4 \%$ & & & $0,1 \%$ \\
\hline & $(n=1)$ & $(n=2)$ & $(n=30)$ & $(n=284)$ & $(n=1)$ & & & $(n=1)$ \\
\hline \multirow[t]{3}{*}{ Innere Medizin } & $79,6 \%$ & $1,4 \%$ & $3,2 \%$ & $0,2 \%$ & $0,3 \%$ & $1,7 \%$ & $0,2 \%$ & $13,3 \%$ \\
\hline & $53,5 \%$ & $4,6 \%$ & $4,0 \%$ & $0,6 \%$ & $1,1 \%$ & $2,1 \%$ & $1,0 \%$ & $7,2 \%$ \\
\hline & $(n=694)$ & $(n=12)$ & $(n=28)$ & $(n=2)$ & $(n=3)$ & $(n=15)$ & $(n=2)$ & $(n=116)$ \\
\hline \multirow[t]{3}{*}{ Kinder- und Jugendmedizin } & $8,6 \%$ & $0,6 \%$ & $1,6 \%$ & $1,0 \%$ & $73,9 \%$ & $0,6 \%$ & & $13,7 \%$ \\
\hline & $2,1 \%$ & $0,8 \%$ & $0,7 \%$ & $0,9 \%$ & $85,9 \%$ & $0,3 \%$ & & $2,7 \%$ \\
\hline & $(n=27)$ & $(n=2)$ & $(n=5)$ & $(n=3)$ & $(n=232)$ & $(n=2)$ & & $(n=43)$ \\
\hline \multirow[t]{3}{*}{$\begin{array}{l}\text { Orthopädische Chirurgie } \\
\text { und Traumatologie } \\
\text { des Bewegungsapparates }\end{array}$} & $2,0 \%$ & & $30,8 \%$ & & & & $63,2 \%$ & $4,0 \%$ \\
\hline & $0,4 \%$ & & $11,2 \%$ & & & & $82,5 \%$ & $0,6 \%$ \\
\hline & $(n=5)$ & & $(n=78)$ & & & & $(n=160)$ & $(n=10)$ \\
\hline \multicolumn{9}{|l|}{ Psychiatrie und } \\
\hline \multirow[t]{3}{*}{ Psychotherapie } & $4,2 \%$ & & $0,5 \%$ & & & $91,0 \%$ & & $4,2 \%$ \\
\hline & $2,1 \%$ & & $0,4 \%$ & & & $79,9 \%$ & & $1,7 \%$ \\
\hline & $(n=27)$ & & $(n=3)$ & & & $(n=579)$ & & $(n=27)$ \\
\hline \multirow[t]{3}{*}{ Nicht entschieden } & $30,3 \%$ & $4,1 \%$ & $20,0 \%$ & $3,8 \%$ & $2,4 \%$ & $10,6 \%$ & $2,6 \%$ & $26,2 \%$ \\
\hline & $7,9 \%$ & $5,3 \%$ & $9,8 \%$ & $3,9 \%$ & $3,0 \%$ & $5,0 \%$ & $4,6 \%$ & $5,5 \%$ \\
\hline & $(n=103)$ & $(n=14)$ & $(n=68)$ & $(n=13)$ & $(n=8)$ & $(n=36)$ & $(n=9)$ & $(n=89)$ \\
\hline \multirow[t]{3}{*}{ Andere } & $12,5 \%$ & $0,6 \%$ & $6,0 \%$ & $0,1 \%$ & $1,1 \%$ & $3,5 \%$ & $0,5 \%$ & $75,7 \%$ \\
\hline & $15,3 \%$ & $3,4 \%$ & $13,5 \%$ & $0,3 \%$ & $6,7 \%$ & $7,6 \%$ & $4,1 \%$ & $74,2 \%$ \\
\hline & $(n=198)$ & $(n=9)$ & $(n=94)$ & $(n=1)$ & $(n=18)$ & $(n=55)$ & $(n=8)$ & $(n=1195)$ \\
\hline
\end{tabular}


Tabelle 8

Mittelwerte für den Kennwert für die sieben Fachrichtungen mit den meisten Assistenzärzten.

\begin{tabular}{lllr} 
Fachrichtung & Mittelwert & $\mathbf{9 5 \%}$ KI & N \\
\hline Chirurgie & 4,01 & $3,93-4,08$ & 641 \\
\hline Anästhesiologie & 4,21 & $4,11-4,31$ & 251 \\
\hline $\begin{array}{l}\text { Orthopädische Chirurgie und Traumatologie } \\
\text { des Bewegungsapparates }\end{array}$ & 4,28 & $4,15-4,41$ & 184 \\
\hline $\begin{array}{l}\text { Gynäkologie } \\
\text { Kinder- und Jugendmedizin }\end{array}$ & 4,32 & $4,23-4,42$ & 314 \\
\hline Innere Medizin & 4,45 & $4,36-4,54$ & 255 \\
\hline Psychiatrie und Psychotherapie & 4,48 & $4,44-4,52$ & 1206 \\
\hline
\end{tabular}

Anmerkung: Die Werte schwanken zwischen 1 «sehr schlechter Kennwert» und 6 «sehr guter Kennwert». Nur die Antworten von Assistenzärzten, die zwei Monate oder länger an der Weiterbildungsstätte tätig waren, wurden berücksichtigt.

Tabelle 9

Der Einfluss der Anzahl Assistenzärzte (AA) an einer Weiterbildungsstätte auf den Kennwert. Mittelwerte für die sieben Fachrichtungen mit den meisten Assistenzärzten.

\begin{tabular}{|c|c|c|c|}
\hline Fachrichtung & $1-3 \mathrm{AA}$ & 4-10 AA & 11 und mehr AA \\
\hline Anästhesiologie & $4,75_{a}(n=20)$ & $4,26_{b}(n=83)$ & $4,11_{b}(n=148)$ \\
\hline Innere Medizin & $4,70_{a}(n=75)$ & $4,48_{b}(n=343)$ & $4,45_{b}(n=788)$ \\
\hline Psychiatrie und Psychotherapie & $4,78(n=32)$ & $4,61(n=163)$ & $4,52(n=481)$ \\
\hline $\begin{array}{l}\text { Orthopädische Chirurgie } \\
\text { und Traumatologie } \\
\text { des Bewegungsapparates }\end{array}$ & $4,42(n=40)$ & $4,06(n=55)$ & $4,35(n=89)$ \\
\hline Gynäkologie und Geburtshilfe & $4,65_{a}(n=49)$ & $4,39_{a}(n=165)$ & $4,04_{b}(n=100)$ \\
\hline Kinder- und Jugendmedizin & $4,58(n=14)$ & $4,34(n=92)$ & $4,51(n=149)$ \\
\hline Chirurgie & $4,01(n=30)$ & $3,99(n=246)$ & $4,01(n=365)$ \\
\hline
\end{tabular}

Anmerkung. Mittelwerte mit unterschiedlichen Buchstaben unterscheiden sich signifikant $(p<0,05)$. Nur die Antworten von Assistenzärzten, die zwei Monate oder länger an der Weiterbildungsstätte tätig waren, wurden berücksichtigt.

Tabelle 11

Mittelwerte für die Attitude für die sieben Fachrichtungen mit den meisten Assistenzärzten.

\begin{tabular}{lllr} 
Fachrichtung & Mittelwert & $\mathbf{9 5 \% ~ K I}$ & N \\
\hline Anästhesiologie & 4,57 & $4,46-4,67$ & 249 \\
\hline Chirurgie & 4,57 & $4,50-4,65$ & 642 \\
\hline $\begin{array}{l}\text { Orthopädische Chirurgie und Traumatologie } \\
\text { des Bewegungsapparates }\end{array}$ & 4,67 & $4,54-4,80$ & 184 \\
\hline Kinder- und Jugendmedizin & 4,76 & $4,66-4,85$ & 258 \\
\hline $\begin{array}{l}\text { Gynäkologie } \\
\text { Innere Medizin }\end{array}$ & 4,80 & $4,71-4,91$ & 315 \\
\hline Psychiatrie und Psychotherapie & 4,86 & $4,82-4,91$ & 1214 \\
\hline
\end{tabular}

Anmerkung: Die Werte schwanken zwischen 1 «sehr schlechte Attitude» und 6 «sehr gute Attitude». Nur die Antworten von Assistenzärzten, die zwei Monate oder länger an der Weiterbildungsstätte tätig waren, wurden berücksichtigt.

\section{Teilzeitstellen}

90,7\% ( $\mathrm{n}=4847)$ der Assistenzärzte gaben an, eine Vollzeitstelle (95\% und mehr) innezuhaben, 3,5\% $(\mathrm{n}=189)$ haben ein Teilzeitpensum zwischen $76 \%$ und $94 \%$ inne und weitere $5,8 \%$ ( $\mathrm{n}=309$ ) haben ein Teilzeitpensum zwischen $50 \%$ und $75 \%$ inne. Es gibt dabei deutliche Unterschiede zwischen den Geschlechtern. Während bei den Männern 96,3\% ( $\mathrm{n}=2702)$ eine Vollzeitstelle innehaben, sind es bei den Frauen $84,4 \%(n=2118)$.

Zusätzlich wurde untersucht, ob es zwischen Teilzeitarbeit und angestrebtem Facharzttitel einen Zusammenhang gibt. Tabelle 12 kann entnommen werden, dass es bei den Frauen einen deutlichen Zusammenhang zwischen Teilzeitstellen und angestrebtem Facharzttitel gibt. Die Weiterbildung in Kinder- und Jugendpsychiatrie sowie Psychiatrie und Psychotherapie wird von den Frauen in weniger als der Hälfte beziehungsweise knapp der Hälfte in Vollzeitarbeit absolviert. In den übrigen Fächern absolvieren weniger als 10\% der Frauen ihre Weiterbildung in Teilzeit. Bei den Männern, welche den Facharzttitel Psychiatrie und Psychotherapie anstreben, absolvieren $15,3 \%(n=49)$ die Weiterbildung in Teilzeit. Bei den anderen Facharzttiteln liegt die Quote der Männer, die eine Teilzeitstelle haben, mehr oder weniger deutlich unter $10 \%$.

\section{Arbeitszeit}

Die Assistenzärzte gaben ihre durchschnittlichen wöchentlichen Arbeitszeiten an. Damit die Zahlen vergleichbar sind, wurden nur die Daten von Assistenzärzten berücksichtigt, welche eine Vollzeitstelle innehatten. Aussreiser nach oben

\section{Tabelle 10}

Durchschnittswerte für den Kennwert bezogen auf die Weiterbildungsstätten.

\begin{tabular}{rcc} 
& Häufigkeiten & Prozente \\
\hline $1-1,5$ & 0 & 0 \\
$>1,5-2$ & 1 & 0,1 \\
$>2-2,5$ & 6 & 0,6 \\
$>2,5-3$ & 17 & 1,7 \\
$>3-3,5$ & 38 & 3,9 \\
$>3,5-4$ & 106 & 10,9 \\
$>4-4,5$ & 257 & 26,4 \\
$>4,5-5$ & 280 & 28,8 \\
$>5-5,5$ & 194 & 20,0 \\
$>5,5-6$ & 73 & 7,5
\end{tabular}

Anmerkung: Analyseeinheiten sind hier die Weiterbildungsstätten. 
(>80 h/Woche) und nach unten $(<44 \mathrm{~h} /$ Woche) wurden eliminiert. Die Daten von 263 (5,8\%) Assistenzärzten wurden deshalb nicht berücksichtigt. Die Durchschnittswerte für die sieben Fachrichtungen mit den meisten Assistentenstellen sind in Tabelle 13 aufgeführt. Zwischen der Umfrage 2003 und der Umfrage 2004 konnten zum Teil deutliche Unterschiede beobachtet werden. Bei allen Fachrichtungen nahm die wöchentliche Arbeitszeit im Vergleich zum Vorjahr ab.

Zusätzlich zur Arbeitszeit wurden die Assistenzärzte auch gefragt, wie viele Stunden theoretischer Weiterbildung (Vorlesungen, Operationskurse, andere Kurse, Seminare) sie im Durchschnitt pro Woche besucht hatten. Die Extremwerte von 11 Stunden oder mehr wurden auf Missing gesetzt. Die Daten von 18 (0,4\%) Assistenzärzten wurden deshalb nicht berücksichtigt. Die Ergebnisse für die Jahre 2003 und 2004 sind in Tabelle 14 aufgeführt. Die Zahl der besuchten Weiterbildungsstunden blieb stabil oder nahm im Vergleich zum Vorjahr sogar zu. Eine signifikante Zunahme konnte für die Fachrichtung Orthopädische Chirurgie und Traumatologie des Bewegungsapparates beobachtet werden.

\section{Eigeninitiative der Assistenzärzte}

Für eine gute Weiterbildung müssen die Assistenzärzte aktiv werden. Mit mehreren Fragen wurde überprüft, ob die Assistenzärzte Eigeninitiative bei der Weiterbildung zeigen (vgl. Tab. 15). Jeder zweite Assistenzarzt möchte eine wissenschaftliche Arbeit publizieren. Deutlich mehr als die Hälfte der Assistenzärzte leistet einen Beitrag zur Weiterbildung an der Weiterbildungsstätte und besucht regelmässig Weiterbildungsveranstaltungen ausserhalb der regulären Arbeitszeit. Eine Minderheit von Assistenzärzten wird kaum aktiv.

Von den Assistenzärzten, welche das Ziel haben, eine wissenschaftliche Arbeit $\mathrm{zu}$ publizieren, haben $68 \%(\mathrm{n}=1796)$ auch Gelegenheit, an einer wissenschaftlichen Publikation zu arbeiten. 32\% ( $\mathrm{n}=846$ ) der Assistenzärzte, welche eine wissenschaftliche Arbeit publizieren möchten, sehen demnach keine Gelegenheit, tatsächlich eine wissenschaftliche Arbeit zu verfassen. Ebenfalls $32 \%(\mathrm{n}=742)$ der Assistenzärzte, die kein Interesse an der Publikation einer wissenschaftlichen Arbeit zeigen, hätten aber Gelegenheit, an einer wissenschaftlichen Publikation zu arbeiten.

Die Bereitschaft zum wissenschaftlichen Publizieren oder der Besuch von externen Weiter- bildungsveranstaltungen wird stark durch den angestrebten Facharzttitel beeinflusst. In Tabelle 16 sind die Antworten für zwei Fragen zur Eigeninitiative für die neun am häufigsten angestrebten Facharzttitel aufgeführt. Assistenzärzte, welche einen Facharzttitel in Chirurgie oder Orthopädischer Chirurgie anstreben, zeigen am meisten, die künftigen Allgemeinmediziner am wenigsten Eigeninitiative. Nur $15 \%$ der Allgemeinmediziner haben das Ziel, wissenschaftliche Arbeiten zu publizieren, und nur jeder zweite besucht regelmässig Weiterbildungsveranstaltungen ausserhalb der regulären Arbeitszeit.

\section{Fehlerkultur}

Die Assistenzärzte beantworteten verschiedene Fragen zur Fehlerkultur. Es handelt sich dabei um die subjektive Wahrnehmung der Fehlerkultur durch die Personen, die in Weiterbildung sind. Auf die Aussage «An unserer Weiterbildungsstätte gibt es ein CIRS (Critical Incidence Reporting System) oder ein anderes System zur Meldung kritischer Ereignisse und Komplikationen» antworteten 41,9\% $(n=2094)$ mit ja, 32,5\% ( $\mathrm{n}=1623)$ mit nein und $25,6 \%(\mathrm{n}=1278)$ der Assistenzärzte mit weiss nicht. Dabei wurden ausschliesslich die Antworten von jenen Assistenzärzten berücksichtigt, die zwei Monate oder länger bei der entsprechenden Weiterbildungsstätte tätig waren. Auf die Aussage «An unserer Weiterbildungsstätte gibt es regelmässig Konferenzen zur Besprechung von Komplikationen und Fehlern» antworteten die Assistenzärzte wie folgt: 39,3\% $(n=1966)$ ja, 50,8\% $(n=2541)$ nein und 10,0\% ( $\mathrm{n}=498)$ weiss nicht. Die Aussage «An unserer Weiterbildungsstätte führen Meldungen von Zwischenfällen dazu, dass die Prozesse verbessert werden» wurde von $61,7 \%$ ( $\mathrm{n}=3080$ ) der Assistenzärzte mit ja, von 16,8\% $(\mathrm{n}=837)$ mit nein und von $21,6 \%(\mathrm{n}=1076) \mathrm{mit}$ weiss nicht beantwortet.

Bei den Antworten auf die Frage, ob es ein CIRS oder ein anderes System zur Meldung kritischer Ereignisse und Komplikationen gibt, gab es fachspezifische Unterschiede (vgl. Tab. 17). Bei den Fachrichtungen Kinder- und Jugendmedizin sowie Anästhesiologie gaben am meisten Befragte an, dass sie über ein System zur Meldung von Fehlern und Komplikationen Bescheid wüssten. Die tiefsten Werte wurden für die Fachrichtung Psychiatrie und Psychotherapie beobachtet. Ebenfalls signifikante Unterschiede zwischen den wichtigsten Fachrichtungen konnte für die Frage, ob es regelmässig Konferenzen zur Besprechung von Komplikationen und Fehlern gebe, beobachtet werden (vgl. Tab. 18). 
Tabelle 12

Assistenzärztinnen und Teilzeitstellen für die am häufigsten angestrebten Facharzttitel.

\begin{tabular}{|c|c|c|c|c|}
\hline & $\begin{array}{l}\text { Vollzeitstelle } \\
\text { (95\% oder mehr) }\end{array}$ & $\begin{array}{l}\text { Teilzeit } \\
\text { (76-94\%) }\end{array}$ & $\begin{array}{l}\text { Teilzeit } \\
(50-75 \%)\end{array}$ & Total \\
\hline Allgemeinmedizin & $90,5 \%(190)$ & $1,0 \%(2)$ & $8,6 \%(18)$ & $100 \%(210)$ \\
\hline Anästhesiologie & $94,3 \%(148)$ & $1,3 \%(2)$ & $4,5 \%(7)$ & $100 \%(157)$ \\
\hline Chirurgie & $100,0 \%(96)$ & & & $100 \%(96)$ \\
\hline Gynäkologie & $92,2 \%(237)$ & & $7,8 \%(20)$ & $100 \%(257)$ \\
\hline Innere Medizin & $91,3 \%(389)$ & $2,1 \%(9)$ & $6,6 \%(28)$ & $100 \%(426)$ \\
\hline Kinder- und Jugendmedizin & $92,1 \%(198)$ & $0,9 \%(2)$ & $7,0 \%(15)$ & $100 \%(215)$ \\
\hline Kinder- und Jugendpsychiatrie & $46,2 \%(55)$ & $17,6 \%(21)$ & $36,1 \%(43)$ & $100 \%(119)$ \\
\hline $\begin{array}{l}\text { Orthopädische Chirurgie } \\
\text { und Traumatologie } \\
\text { des Bewegungsapparates }\end{array}$ & $100,0 \%(36)$ & & & $100 \%(36)$ \\
\hline Psychiatrie und Psychotherapie & $57,6 \%(181)$ & $21,7 \%(68)$ & $20,7 \%(65)$ & $100 \%(314)$ \\
\hline
\end{tabular}

Tabelle 13

Mittelwerte für die durchschnittliche wöchentliche Arbeitszeit 2003 und 2004 der Assistenzärzte mit Vollpensum für die sieben Fachrichtungen mit den meisten Assistenzärzten.

\begin{tabular}{|c|c|c|c|c|c|c|}
\hline & 2003 & & & 2004 & & \\
\hline Fachrichtung & M & $95 \% \mathrm{KI}$ & $\mathrm{N}$ & M & $95 \% \mathrm{KI}$ & $\mathrm{N}$ \\
\hline Chirurgie & 59,85 & $59,30-60,40$ & 587 & 58,87 & $58,31-59,44$ & 585 \\
\hline $\begin{array}{l}\text { Orthopädische Chirurgie } \\
\text { und Traumatologie } \\
\text { des Bewegungsapparates }\end{array}$ & 61,19 & $60,18-62,21$ & 175 & 57,70 & $56,84-58,55$ & 174 \\
\hline Gynäkologie und Geburtshilfe & 57,84 & $57,14-58,53$ & 285 & 56,87 & $56,11-57,63$ & 264 \\
\hline Innere Medizin & 57,04 & $56,71-57,37$ & 1100 & 56,07 & $55,73-56,40$ & 1102 \\
\hline Kinder- und Jugendmedizin & 56,85 & $56,12-57,57$ & 217 & 54,80 & $54,14-55,46$ & 232 \\
\hline Anästhesiologie & 54,41 & $53,87-54,95$ & 224 & 53,80 & $53,25-54,35$ & 232 \\
\hline Psychiatrie und Psychotherapie & 52,78 & $52,28-53,28$ & 454 & 51,85 & $51,38-52,32$ & 440 \\
\hline
\end{tabular}

\section{Tabelle 14}

Mittelwerte für die durchschnittliche theoretische Weiterbildung pro Woche 2003 und 2004 der Assistenzärzte mit Vollpensum für die sieben Fachrichtungen mit den meisten Assistenzärzten.

\begin{tabular}{|c|c|c|c|c|c|c|}
\hline \multirow[b]{2}{*}{ Fachrichtung } & \multicolumn{3}{|l|}{2003} & \multicolumn{3}{|l|}{2004} \\
\hline & M & $95 \% \mathrm{KI}$ & $\mathrm{N}$ & M & $95 \% \mathrm{KI}$ & $\mathrm{N}$ \\
\hline Psychiatrie und Psychotherapie & 4,49 & $4,31-4,68$ & 490 & 4,46 & $4,27-4,64$ & 481 \\
\hline Kinder- und Jugendmedizin & 2,42 & $2,23-2,61$ & 228 & 2,48 & $2,31-2,65$ & 237 \\
\hline Innere Medizin & 2,61 & $2,52-2,70$ & 1144 & 2,69 & $2,60-2,78$ & 1143 \\
\hline $\begin{array}{l}\text { Orthopädische Chirurgie } \\
\text { und Traumatologie } \\
\text { des Bewegungsapparates }\end{array}$ & 2,05 & $1,82-2,28$ & 182 & 2,73 & $2,46-3,00$ & 182 \\
\hline Gynäkologie und Geburtshilfe & 1,72 & $1,56-1,87$ & 295 & 1,82 & $1,67-1,98$ & 282 \\
\hline Anästhesiologie & 1,69 & $1,56-1,82$ & 235 & 1,90 & $1,75-2,04$ & 235 \\
\hline Chirurgie & 1,78 & $1,67-1,89$ & 619 & 1,92 & $1,81-2,02$ & 618 \\
\hline
\end{tabular}


Tabelle 15

Antworten auf verschiedene Fragen zur Eigeninitiative der Assistenzärzte.

\begin{tabular}{llc} 
& nein & ja \\
\hline Haben Sie das Ziel, wissenschaftliche Arbeiten zu publizieren? & $49,1 \%(2453)$ & $50,9 \%(2546)$ \\
\hline $\begin{array}{l}\text { Haben Sie Gelegenheit, an einer wissenschaftlichen Publikation zu arbeiten? } \\
\text { Leisten Sie einen Beitrag zur Weiterbildung an Ihrer Weiterbildungsstätte }\end{array}$ & $46,8 \%(2329)$ & $53,2 \%(2646)$ \\
$\begin{array}{l}\text { (z. B. Gestaltung einer Weiterbildungsveranstaltung für andere Assistenz- } \\
\text { ärzte)? }\end{array}$ & $25,6 \%(1283)$ & 74,4\%(3722) \\
$\begin{array}{l}\text { Besuchen Sie regelmässig Weiterbildungsveranstaltungen ausserhalb der } \\
\text { regulären Arbeitszeit? }\end{array}$ & $39,2 \%(1961)$ & $60,8 \%(3047)$ \\
$\begin{array}{l}\text { Anmerkung. Nur die Antworten von Assistenzärzten, die zwei Monate oder länger an der Weiterbildungsstätte } \\
\text { tätig waren, wurden berücksichtigt. }\end{array}$ &
\end{tabular}

\section{Tabelle 16}

Zeilenprozente für zwei Fragen zur Eigeninitiative der Assistenzärzte für die neun am häufigsten angestrebten Facharzttitel.

\begin{tabular}{|c|c|c|c|c|}
\hline & \multicolumn{2}{|c|}{$\begin{array}{l}\text { Haben Sie das Ziel, wissenschaftliche } \\
\text { Arbeiten zu publizieren? }\end{array}$} & \multicolumn{2}{|c|}{$\begin{array}{l}\text { Besuchen Sie regelmässig } \\
\text { Weiterbildungsveranstaltungen } \\
\text { ausserhalb der regulären } \\
\text { Arbeitszeit? }\end{array}$} \\
\hline & Nein & Ja & Nein & ja \\
\hline Allgemeinmedizin & $346(84,8 \%)$ & $62(15,2 \%)$ & $204(50 \%)$ & $204(50 \%)$ \\
\hline Anästhesiologie & $190(62,3 \%)$ & $115(37,7 \%)$ & $158(51,8 \%)$ & $147(48,2 \%)$ \\
\hline Chirurgie & $93(30,4 \%)$ & $213(69,6 \%)$ & $85(27,5 \%)$ & $224(72,5 \%)$ \\
\hline Gynäkologie & $191(63,7 \%)$ & $109(36,3 \%)$ & $65(21,7 \%)$ & $235(78,3 \%)$ \\
\hline Innere Medizin & $410(50,8 \%)$ & $397(49,2 \%)$ & $423(52,4 \%)$ & $384(47,6 \%)$ \\
\hline Kinder- und Jugendmedizin & $157(52,5 \%)$ & $142(47,5 \%)$ & $171(57,2 \%)$ & $128(42,8 \%)$ \\
\hline Kinder- und Jugendpsychiatrie & $104(72,2 \%)$ & $40(27,8 \%)$ & $41(28,7 \%)$ & $102(71,3 \%)$ \\
\hline $\begin{array}{l}\text { Orthopädische Chirurgie } \\
\text { und Traumatologie } \\
\text { des Bewegungsapparates }\end{array}$ & $62(26,5 \%)$ & $172(73,5 \%)$ & $52(22,2 \%)$ & $182(77,8 \%)$ \\
\hline Psychiatrie und Psychotherapie & $364(60,2 \%)$ & $241(39,8 \%)$ & $142(23,5 \%)$ & $461(76,5 \%)$ \\
\hline
\end{tabular}

Anmerkung. Nur die Antworten von Assistenzärzten, die zwei Monate oder länger an der Weiterbildungsstätte tätig waren, wurden berücksichtigt.

Tabelle 17

Antworten und Zeilenprozente für die Aussage «An unserer Weiterbildungsstätte gibt es ein CIRS (Critical Incidence Reporting System) oder ein anderes System zur Meldung kritischer Ereignisse und Komplikationen».

\begin{tabular}{|c|c|c|c|c|}
\hline & Ja & Nein & Weiss nicht & Total \\
\hline Anästhesiologie & $60,6 \%(151)$ & $22,1 \%(55)$ & $17,3 \%(43)$ & $100 \%(249)$ \\
\hline Chirurgie & $48,8 \%(312)$ & $32,0 \%(205)$ & $19,2 \%(123)$ & $100 \%(640)$ \\
\hline Gynäkologie & $46,5 \%(146)$ & $28,3 \%(89)$ & $25,2 \%(79)$ & $100 \%(314)$ \\
\hline Innere Medizin & $41,3 \%(499)$ & $31,2 \%(377)$ & $27,5 \%(332)$ & $100 \%(1208)$ \\
\hline Kinder- und Jugendmedizin & $70,8 \%(182)$ & $16,0 \%(41)$ & $13,2 \%(34)$ & $100 \%(257)$ \\
\hline $\begin{array}{l}\text { Orthopädische Chirurgie } \\
\text { und Traumatologie } \\
\text { des Bewegungsapparates }\end{array}$ & $39,9 \%(73)$ & $32,8 \%(60)$ & $27,3 \%(50)$ & $100 \%(183)$ \\
\hline Psychiatrie und Psychotherapie & $27,4 \%(186)$ & $38,1 \%(258)$ & $34,5 \%(234)$ & $100 \%(678)$ \\
\hline
\end{tabular}




\section{Diskussion}

Die subjektive Beurteilung der Weiterbildung durch die Assistenzärzte ist eines der Elemente einer Qualitätskontrolle in der Weiterbildung. Eine Umfrage bei den Leitern der Weiterbildungsstätten zeigte, dass eine Mehrheit die strukturierten Rückmeldungen als fair und akzeptabel einstufte [7]. Obwohl der Fragebogen 2003 insgesamt positiv beurteilt wurde, wurde dieser für die Umfrage 2004 weiter verbessert. Die zahlreichen Anregungen und Wünsche der Leiter und Leiterinnen von Weiterbildungsstätten wurden so gut wie möglich berücksichtigt.

Der Rücklauf der Umfrage 2004 ist ähnlich hoch ausgefallen wie im Vorjahr und liegt über oder im Rahmen vergleichbarer Studien im Ausland $[8,9]$. Insgesamt liefern die Daten der Umfrage 2004 ein aktuelles und umfassendes Bild über die Weiterbildung aus Sicht der Assistenzärzte.

Vergleicht man die Ergebnisse der Umfragen 2003 und 2004, so sind zwei Trendresultate auffällig. In der Fachrichtung Psychiatrie und Psychotherapie hat der Frauenanteil nochmals zugenommen. Der Anteil der Assistenzärzte mit deutschem Staatsexamen hat 2004 im Vergleich zum Vorjahr um 10\% zugenommen. Jeder vierte Assistenzarzt in Weiterbildung hat das Staatsexamen in Deutschland gemacht.

Der Kennwert liegt für die chirurgischen Weiterbildungsstätten am tiefsten. Dies ist wohl auf die hohe Arbeitszeit und den hohen Anteil von fachfremden Assistenzärzten zurückzuführen. Letztere erhalten leider immer noch oft keine dem Weiterbildungsziel angepasste Weiterbildung.

Die von den Assistenzärzten angegebene wöchentliche Arbeitszeit war bei allen wichtigen
Fachrichtungen tiefer als im Vorjahr. Bei den Assistenzärzten in der orthopädischen Chirurgie reduzierte sich die durchschnittliche Arbeitszeit um über drei Stunden und bei den Assistenzärzten in der Kinder- und Jugendmedizin reduzierte sich die Arbeitszeit durchschnittlich um 2 Stunden. Bei den anderen Fachrichtungen fiel die Arbeitszeitreduktion bescheidener aus. Um die Vorschrift der maximalen wöchentlichen Arbeitszeit von 50 Stunden zu erfüllen, muss die Arbeitszeit im Jahr 2005 an den psychiatrischen Weiterbildungsstätten durchschnittlich um zwei, an den chirurgischen Weiterbildungsstätten um neun Stunden reduziert werden. Die für die theoretische Weiterbildung aufgewendeten Stunden blieben bei den meisten Fachrichtungen stabil. Im Vergleich zum Vorjahr besuchten die Assistenzärzte der orthopädischen Chirurgie eine zusätzliche halbe Stunde Weiterbildung pro Woche. Die reduzierte Arbeitszeit hat also erfreulicherweise nicht dazu geführt, dass weniger Stunden theoretischer Weiterbildung besucht werden.

Ein mehr oder weniger grosser Anteil der Weiterbildung erfolgt ausserhalb der regulären Arbeitszeit. Dabei konnten erhebliche Unterschiede zwischen den Fachrichtungen beobachtet werden. Assistenzärzte, welche einen Facharzttitel in Chirurgie oder Orthopädischer Chirurgie anstreben, müssen Operationskurse und zum Teil Notfallkurse ausserhalb der Arbeitszeit besuchen. Auch Assistenzärzte, die einen Facharzttitel Kinder- und Jugendpsychiatrie oder Psychiatrie und Psychotherapie anstreben, besuchen Lehrtherapien oder Weiterbildungskurse überdurchschnittlich häufig ausserhalb der regulären Arbeitszeit.

Die Fehlerkultur wurde in der Umfrage 2004 mit ganz konkreten Fragen zum Umgang mit
7 Siegrist M, Giger M. Assistentenund Assistentinnenumfrage 2003 aus Sicht der Leiter und Leiterinnen von Weiterbildungsstätten. Schweiz Ärztezeitung 2004; 85(42):2262-6.

8 Delva MD, Kirby JR, Knapper CK, Birtwhistle RV. Postal survey of approaches to learning among Ontario physicians: implications for continuing medical education. BMJ 2002;325:1218.

9 Keitz SA, Holland GJ, Melander EH, Bosworth HB, Pincus SH. The Veterans Affairs Learners' Perceptions Survey: the foundation for educational quality improvement. Acad Med 2003;78:910-7.

Tabelle 18

Antworten und Zeilenprozente für die Aussage «An unserer Weiterbildungsstätte gibt es regelmässig Konferenzen zur Besprechung von Komplikationen und Fehlern».

\begin{tabular}{lllll} 
& Ja & Nein & Weiss nicht & Total \\
\hline Anästhesiologie & $43,6 \%(109)$ & $48,0 \%(120)$ & $8,4 \%(21)$ & $100 \%(250)$ \\
\hline Chirurgie & $48,1 \%(307)$ & $43,3 \%(276)$ & $8,6 \%(55)$ & $100 \%(638)$ \\
\hline $\begin{array}{l}\text { Gynäkologie } \\
\text { Innere Medizin }\end{array}$ & $51,7 \%(163)$ & $42,5 \%(134)$ & $5,7 \%(18)$ & $100 \%(315)$ \\
\hline Kinder- und Jugendmedizin & $48,9 \%(350)$ & $59,3 \%(718)$ & $11,7 \%(142)$ & $100 \%(1210)$ \\
\hline $\begin{array}{l}\text { Orthopädische Chirurgie } \\
\text { und Traumatologie } \\
\text { des Bewegungsapparates }\end{array}$ & $49,5 \%(91)$ & $39,7 \%(73)$ & $9,3 \%(24)$ & $100 \%(258)$ \\
\hline \begin{tabular}{l} 
Psychiatrie und Psychotherapie \\
\hline
\end{tabular} & $40,6 \%(276)$ & $49,8 \%(338)$ & $9,6 \%(65)$ & $100 \%(184)$ \\
\hline
\end{tabular}

Anmerkung. $\chi_{(12)}=117,06, p<0,001$. Nur die Antworten von Assistenzärzten, die zwei Monate oder länger an der Weiterbildungsstätte tätig waren, wurden berücksichtigt. 
Fehlern und Komplikationen gemessen. Die Daten lassen nur einen Schluss zu: Der Umgang mit Fehlern und Komplikationen sollte einen grösseren Stellenwert in der Weiterbildung erhalten. Als erstes sollte die Kommunikation verbessert werden, um den Anteil der Assistenten, welche keine Kenntnis über das Vorhandensein von Meldesystem oder Fehlerkonferenzen angeben, zu reduzieren. Dabei sind die fachspezifischen Unterschiede zu beachten. Am besten ist die Fehlerkultur in Pädiatrie und Anästhesiologie eingeführt. Bei der Inneren Medizin gaben nur 30\% der Befragten an, dass es an ihrer Weiterbildungsstätte regelmässig Konferenzen zur Besprechung von Komplikationen und Fehlern gibt. Bezüglich der systematischen Erfassung von kritischen Ereignissen und Fehlern schnitt die Psychiatrie und Psychotherapie relativ schlecht ab. Nur gerade $27 \%$ der Befragten aus dieser Fachrichtung gaben an, dass an ihrer Weiterbildungsstätte ein solches System vorhanden ist.

Bei den vorliegenden Ergebnissen handelt es sich um die subjektive Einschätzung der Assistenzärzte. Es lässt sich einzig abschätzen, wie viele Assistenzärzte von einem solchen System zur Meldung kritischer Ereignisse und Komplikationen Kenntnis haben. Auf Grund der vorliegenden Daten können wir nicht sagen, an wie vielen Weiterbildungsstätten tatsächlich ein System zur Erfassung von Fehlern oder Beinahefehlern vorhanden ist.

In der Psychiatrie zeichnet sich ein Engpass ab: Einerseits hat sich die relative Zahl der Assistenzärzte, welche den Titel Psychiatrie und Psychotherapie anstreben, innerhalb von fünf Jahren halbiert und anderseits steigt der Frauenanteil weiterhin stark, und nur knapp die Hälfte der Frauen absolvieren die Weiterbildung in Vollzeitarbeit. Die beobachtete Tendenz könnte etwas abgeschwächt werden, wenn die Assistenzärzte, die sich noch nicht für einen Facharzttitel entschieden haben, überdurchschnittlich häufig Psychiatrie wählen würden.

\section{Ausblick}

Der Fragebogen muss von Assistenzärzten, welche einen von 43 Facharzttiteln anstreben, aus- gefüllt werden können. Die Fragen können deshalb nicht zu spezifisch sein, sondern müssen einen gewissen Abstraktionsgrad aufweisen. Der Vorteil ist, dass dadurch Vergleiche zwischen den Fachrichtungen möglich werden. Ein grosser Nachteil ist aber, dass spezifische Fragen, welche für bestimmte Fachrichtungen sehr wichtig sein könnten, fehlen. Diese Problematik soll bei der Umfrage 2005 teilweise entschärft werden. Die Fachgesellschaften mit den meisten Assistenzärzten erhielten die Möglichkeit, individuelle Fragen zu stellen, welche für ihre Fachgesellschaft von besonderem Interesse sind. Interesse an einem solchen fachspezifischen Modul wurde von den Fachgesellschaften Innere Medizin und Orthopädische Chirurgie und Traumatologie des Bewegungsapparates bekundet. Bei der Ausarbeitung des Moduls wird von den entsprechenden Fachgesellschaften eine aktive Mitarbeit erwartet.

Die Zahl der ausgefüllten Fragebogen nahm bei der Umfrage leicht zu, aber auch die Zahl der gemeldeten Assistenzstellen war etwas höher als im Vorjahr. Die Rücklaufquote der Umfrage 2004 ist deshalb vergleichbar mit der Rücklaufquote der Umfrage 2003. Das Ziel, den Rücklauf zu erhöhen, wurde leider nicht erreicht. Die Fragebogen werden an die Leiter und Leiterinnen der Weiterbildungsstätten geschickt, und diese verteilen die Fragebogen an die Assistenzärzte. Deshalb können keine gezielten Mahnungen an die Assistenzärzte versandt werden. Ein Obligatorium für das Ausfüllen des Fragebogens, wie es verschiedentlich vorgeschlagen wurde, lässt sich mit diesem Vorgehen nicht durchsetzen. Eine mögliche deutlich aufwendigere Lösung zur «Vollerfassung» könnte dereinst in der Kennzeichnung der Fragebogen mit der EAN-Nummer der Assistenzärzte auf Grund eines eidgenössischen Weiterbildungsregisters bestehen. Es ist auch in nächster Zukunft wichtig, dass die Leiter und Leiterinnen von Weiterbildungsstätten ihre Mitarbeiter zu einer Teilnahme motivieren. Wenn die Assistenzärzte wissen, dass die Resultate für die Weiterbildner wichtig sind und dass sie an den Resultaten interessiert sind, so kann mit einem grösseren Rücklauf gerechnet werden, und die Assistenzärzte füllen den Fragebogen mit der notwendigen Ernsthaftigkeit aus. 